\title{
Management of Patients with Hernia or Incisional Hernia Undergoing Surgery for Morbid Obesity
}

\author{
Ramon Vilallonga, ${ }^{1}$ José Manuel Fort, ${ }^{1}$ Oscar Gonzalez, ${ }^{1}$ Juan Antonio Baena, ${ }^{1}$ \\ Albert Lecube, ${ }^{2}$ and Manuel Armengol ${ }^{1}$ \\ ${ }^{1}$ General Surgery Department, Universitary Hospital Vall d'Hebron, Passeig de la Vall d'Hebron, 119-129, 08035 Barcelona, Spain \\ ${ }^{2}$ Obesity Unit, Endocrine Department, Universitary Hospital Vall d'Hebron, Passeig de la Vall d'Hebron, 119-129, \\ 08035 Barcelona, Spain
}

Correspondence should be addressed to Ramon Vilallonga, vilallongapuy@hotmail.com

Received 19 July 2010; Accepted 26 October 2010

Academic Editor: Michael M. Murr

Copyright (C) 2011 Ramon Vilallonga et al. This is an open access article distributed under the Creative Commons Attribution License, which permits unrestricted use, distribution, and reproduction in any medium, provided the original work is properly cited.

\begin{abstract}
Morbidly obese patients (MOPs) are predisposed to developing abdominal wall hernias with the potential complication of small bowel obstruction and other morbidity. We report our experience in treating morbidly obese patients. Hernia prophylaxis has been attempted as a means of decreasing the incisional hernia risk associated with weight loss surgery. The controversy regarding the optimal time and method of repair of abdominal wall hernias in patients undergoing open or laparoscopic gastric bypass is discussed with emphasis placed on either a simultaneous repair or splits of the omentum, and of leaving a plug in the hernia defect, to allow time to perform a delayed repair.
\end{abstract}

\section{Introduction}

Morbidly obese patients (MOPs) are predisposed to developing abdominal wall hernias with the potential complication of small bowel obstruction and other morbidity [1]. We report our experience in treating morbidly obese patients. Hernia prophylaxis has been attempted as a means of decreasing the incisional hernia risk associated with weight loss surgery [2]. The controversy regarding the optimal time and method of repair of abdominal wall hernias in patients undergoing open or laparoscopic gastric bypass is discussed with emphasis placed on either a simultaneous repair or split of the omentum, and of leaving a plug in the hernia defect, to allow time to perform a delayed repair [3].

\section{Methods}

Medical records of consecutive morbidly obese patients who underwent open or laparoscopic Roux-en-Y (ORYGBPLRYGBP) gastric bypass with a secondary diagnosis of ventral hernia were reviewed. Only patients who were beyond 12 months of followup were included.
In this study, all details of consecutive patients who underwent ORYGBP or LRYGBP at the University Hospital Vall d'Hebron, from May 2001 to February 2010 were entered into an electronic database. The medical charts of these patients were reviewed. The data that was obtained included demographics, body mass index (BMI), and hernia characteristics such as status of natural history (reduced versus incarcerated). Operative details included the hernia management. Short-and long-term followup data consisted of length of hospital stay, the incidence of early and late complications, length of followup, and the frequency of recurrence by clinical examination. The BMI, a standard index for classifying obesity, was calculated as BMI = weight $(\mathrm{kg}) /$ height $2(\mathrm{~m})$. Data are presented as mean and range.

\section{Results}

The study population was 398 patients, 41 of who had ventral hernias or incisional hernias (Table 1). There were six groups of patients according to the method of repair: primary repair before the ORYGBP or LRYGBP (10 patients), 
TABLE 1: Characteristics of the study group of patients.

\begin{tabular}{ll}
\hline Mean age (years) & $44(19-64)$ \\
Male/Female & $113 / 285$ \\
Weight $(\mathrm{Kg})$ & $129(82-194)$ \\
Weight excess $(\mathrm{Kg})$ & $66(39-100)$ \\
BMI $\left(\mathrm{Kg} / \mathrm{m}^{2}\right)$ & $49(31-71)$ \\
$\%$ of superobese patients (IMC $>50)$ & 40 \\
Age of inicial overweight (years) & $22(7-54)$ \\
\hline
\end{tabular}

primary repair without mesh (6 patients), primary repair with mesh ( 3 patients), deferred treatment (20 patients), and finally a group of patients in whom the hernia was reduced but not repaired for technical reasons (4 patients). Average follow-up was 46 months. There was a $34 \%$ of recurrence in the primary repair group. Two of the patients in the deferred treatment and desincarcerated group (40\%) presented with small bowel obstruction due to incarceration (Table 2).

From the groups of patients with primary repair without mesh or the group with primary repair with mesh during the surgery, we had a $2 / 6(33 \%)$ and $1 / 3(33 \%)$ of patients with incisional hernia, respectively. All these patients were treated with ORYGBP. Of these patients, after weight loss, all three patients had their incisional hernia repaired. None of the patients complained about obstructive symptoms after ORYBGP.

In 17 patients, we deferred treatment of the hernia or incisional hernia. In this group, none of the patients had occlusive symptoms, and hernia or incisional hernia treatment was performed during the plastic surgery treatment, after weight loss.

In a small number of patients $(n=5)$, the hernia was reduced but not repaired during surgery. Because of technical constraints, we had to reduce the content of the sac (with omentum inside) without subsequent repair of the fascial defect. In two patients (40\%), the food loop of the Y de Roux was incarcerated, producing a picture of intestinal obstruction that required urgent surgery.

\section{Discussion}

MOPs have a greater chance to develop abdominal wall hernias and their potential complication such as small bowel obstruction [1]. New modern hernia repair approach has increased the opportunities to treat patients in a one-stage procedure in order to reduce the risk of incisional hernias [2]. This has become possible thanks to progress in the surgical approach to hernias with application of minimally invasive surgical techniques. However, the controversy regarding the optimal time and method of repair of abdominal wall hernias in patients undergoing open or laparoscopic gastric bypass is still discussed [3]. Also, a better understanding of how to apply this to reconstructing abdominal wall defects and the new synthetic meshes with a better understanding of the mechanical properties necessary to secure hernia repair, with newer biomaterials that provide for tissue ingrowth and may be more resistant to infection than traditional meshes, have given a new vista to the treatment of the hernia [4]. This has allowed for opportunities to repair hernias during potentially contaminated operations that may have otherwise required a second operation, such as ORYGBP or LRYGBP.

Morbidly obese patients are predisposed to developing abdominal wall hernias [1] and these are a common cause of morbidity and mortality [3]. Overweight is an important issue for incisional hernias and for these reasons many patients have already undergone a hernia repair surgery before the bariatric surgery [3].

Incarceration of such hernias can lead to small bowel obstruction (SBO). In addition, the resultant derangement of the GI anatomy after gastric bypass increases the incidence, and level of difficulty in making a diagnosis of partial SBO [5]. There is no clear consensus among bariatric surgeons on the optimal time and method of repair of abdominal wall hernias in patients undergoing laparoscopic Roux-en$\mathrm{Y}$ gastric bypass (LRYGBP). The management of primary and incisional abdominal wall hernias continues to evolve from the early days of primary hernia repair. Lastly, there has been a return to primary tissue repairs using components separation technique, augmented with mesh as necessary. This combination of education and new materials such as lightweight meshes has provided the surgeon with a basis of performing a better hernia repair [6].

Better appreciation of patient characteristics may help to support which type of procedure or mesh use will likely succeed. Risk factors for the development of abdominal wall incisional hernias may include the following: overweight, smoking, age greater than 60 years, wound infection, relaparotomy, chronic medical conditions (such as cirrhosis or cardiopulmonary disease), and chronic steroid use. The relative ratio and amount of type I collagen may determine patients at risk [7]. Also, obesity is a risk factor for ventral hernias, both as a feature of their occurrence and as a factor in recurrence after repair [8]. Common in the United States, obesity is associated with increased risk of other medical problems. Approximately $63 \%$ of men and $55 \%$ of women aged 25 years or older are overweight or obese [9]. According to some studies, morbid obesity, defined as a body mass index (BMI) exceeding 40, is a major risk factor in the development of incisional hernia, with $20 \%$ to $28 \%$ of obese patients who undergo abdominal surgery developing an incisional hernia within 12 to 28 months of the initial procedure $[10,11]$. Increased abdominal pressure, as seen in obesity, particularly in those individuals with large amounts of central adiposity, is associated with a predisposition to umbilical and incisional hernias. In our series, abdominal wall pathology is found in $11 \%$ of the patients who underwent to surgery. Also, our series confirms the high recurrence rate among these patients, which amounts to $25 \%$. In fact, Sugerman et al. have stressed that obesity may be a greater risk factor for incisional hernia occurrence than chronic steroid use [12]. This is particularly true for patients with sleep apnea or the obesity hypoventilation syndrome, factors that proved to be significantly associated with ventral hernia formation [13]. As we witness the relentless increase in the prevalence of obesity, we can only expect a coincident rise in the incidence of ventral hernias, with attendant potentially 
TABLE 2: Characteristics of the patients with hernia or incisional hernia.

\begin{tabular}{lc}
\hline Number of patients & 41 \\
Mean age (years) & $45(22-63)$ \\
Male/Female & $6 / 35$ \\
Weight $(\mathrm{Kg})$ & $134(95-175)$ \\
BMI $\left(\mathrm{Kg} / \mathrm{m}^{2}\right)$ & $48(32-55)$ \\
Groups of treatment: & $10(24,4 \%)$ \\
$\quad$ Hernias repared before ORYBBP or LRYGB & $6(14,6 \%)$ \\
Primary repair without mesh & $3(7,3 \%)$ \\
Primary repair with mesh & $17(41,5 \%)$ \\
Deferred treatment of the hernia or incisional hernia & $5(12,2 \%)$ \\
Hernia was desincarcerated for technical problems but not repaired & $8(19,5 \%)$ \\
Type or location of hernia: & $8(19,5 \%)$ \\
Incisional hernia & $21(51,2 \%)$ \\
Recurrent incisional & $2(4,9 \%)$ \\
Umbilical & $2(4,9 \%)$ \\
Recurrent umbilical & \\
Epigastric &
\end{tabular}

life-threatening complications that may arise from small bowel incarceration. With improved recognition of these problems, surgeons are able to determine extent and timing of treatment, in order to minimize future patient morbidity.

Many authors have suggested that a weight loss may help to improve the technical circumstances of the operation and help to reduce the potential recurrence rate, though it may not change the risk of perioperative complications [14]. However, expectation of weight loss and the time necessary for significant weight loss need to be balanced with the clinical indication for the hernia repair and also for the clinical indication for the bariatric surgery.

Another controversial end-point in bariatric surgery is the hernias identified at the time of surgery. Concerns exist about the risk of mesh infection in relationship to opened bowel at the time of gastric bypass surgery. For this reason, some surgeons consider that hernia is not amenable to primary repair and repair should be deferred until a later time. The use of laparoscopic Ventral Hernia Repair (LVHR) for morbidly obese individuals with large recurrent incisional hernias or small bowel obstruction at presentation and the use of LVHR in combination with LGB certainly qualify as some of those challenging and complex scenarios for the use of LVHR [15].

As in our experience, there is a certain risk that some patients with deferred repair developed a small bowel obstruction, and even patients who underwent a primary repair developed a recurrent hernia. To solve these problems some authors have suggested the use of a biologically derived mesh and found no recurrences in short-term follow up (13 months average) and no mesh infections [13]. Also, the quality of the suture technique is very important in overweight patients, and by focusing on the technique, a reasonable herniation rate can be achieved.

According to our experience, hernias that have to be reduced during the surgery, because of technical needs, should be repaired. Also, the characteristics of the hernia such as smaller necked, deeper hernias, with higher potential for incarceration, may be repaired primarily, accepting a higher recurrence rate to offset the risk of a mesh infection. In contrast, large, shallow defects can be deferred for later repair, while monitoring the patient for clinical symptoms related to the hernia. To our knowledge, there are no prospective studies evaluating the natural history of chronically incarcerated hernias that are discovered at the time of surgery and are then left undisturbed. For these reasons, we do think it can be safer to perform the bariatric procedure with its lost of weight before undergoing an hernia repair. If the hernia is located in a place that requires its desincarceration, it should be repaired during the same bariatric surgery. If not, a definitive plastic surgery after weight lost will be suitable.

Another controversial end-point in hernia repair during bariatric surgery is hernia prophylaxis [16]. This concept was based upon some success from when the mesh was used to repair parastomal hernias. These studies did not result in a significant increase in major wound infections requiring mesh removal [17]. According to the technique, despite good results in terms of safety and minimal recurrence ensured by laparoscopy in the management of incisional hernias, the use of minimally invasive techniques for large incisional wall defects is still controversial.

Further objective studies will need to be done in order to augment this supposition but also to elucidate if the hernia should be repaired during the bariatric procedure, or when the patient has undergone some weight loss or even at the time of abdominal wall plastic surgery, if planned.

There is a great concern among bariatric surgeons about the rational treatment of hernias in the postoperative period. At this stage some issues have to be considered. The first category of patients are those who develop an asymptomatic or minimally symptomatic hernia in the first 
1 to 2 years after surgery and who are planning to undergo abdominoplasty or other types of body-contouring surgery. In these cases, we do consider that hernia repair may be combined with their plastic surgery so that the patients need only undergo one anesthetic course. In addition, there may be significant laxity to the abdominal fascia, which may be reapproximated in a two-layer imbricating fashion that is typical of an abdominoplasty. If the defect is too large for a tension-free repair, mesh should be used as either an onlay or an underlay or repaired using a components separation technique. The existing experience when performing concomitant LVHR and LRYGBP and the use of biomaterials for the morbidly obese population have been controversial [18]. However, some studies have showed that following obesity surgery, simultaneous ventral hernia repair and panniculectomy can be accomplished safely with short hospital stays and few in-hospital complications, and this is independent of the mesh material used [19]. Postoperative wound problems are not infrequent but can be managed in the outpatient setting. Overall, the hernia repair may be combined with the plastic surgery without significant increased risk [20].

The second category is the patient who presents either acutely or has symptoms related to an incisional hernia. These patients should undergo repair in a relatively timely fashion, utilizing a laparoscopic technique if this is suitable to the skill of the surgeon and the complexity of the hernia repair.

\section{Conclusion}

Hernias and abdominal wall incisional hernias are more prevalent in patients with morbid obesity. Hernia development has a significant impact on the obese/postbariatric surgery patient. Diligence in the clinical exam will minimize unanticipated problems in handling large hernias at the time of bariatric procedures. New mesh products and/or components separation techniques can allow for combining procedures so the patients will have the optimum recovery from their operation and the best chance for succeeding at their weight loss operation. In addition, in our experience, repair is not advisable due to its high rate of recurrence. For this reason, it is recommended that a posterior repair with or without dermolipectomy aesthetics is carried out. Abdominal wall hernias unrepaired during surgery will rarely give postoperative complications. However, if a hernia is reduced with omental content and is not repaired, the risk of occlusion is high in the immediate postoperative period according to our experience.

\section{Acknowledgment}

The authors wish to thank Dr. Ramon Vilallonga Foundation for supporting the study. (http://www.fundacioramonvilallonga.org/).

\section{References}

[1] T. Anthony, P. C. Bergen, L. T. Kim et al., "Factors affecting recurrence following incisional herniorrhaphy," World Journal of Surgery, vol. 24, no. 1, pp. 95-101, 2000.

[2] T. Datta, G. Eid, N. Nahmias, and R. M. Dallal, "Management of ventral hernias during laparoscopic gastric bypass," Surgery for Obesity and Related Diseases, vol. 4, no. 6, pp. 754-757, 2008.

[3] M. Sukeik, B. Alkari, and B. J. Ammori, "Abdominal wall hernia during laparoscopic gastric bypass: a serious consideration," Obesity Surgery, vol. 17, no. 6, pp. 839-842, 2007.

[4] M. E. Franklin Jr., J. J. Gonzalez Jr., and J. L. Glass, "Use of porcine small intestinal submucosa as a prosthetic device for laparoscopic repair of hernias in contaminated fields: 2-year follow-up," Hernia, vol. 8, no. 3, pp. 186-189, 2004.

[5] A. Iannelli, E. Facchiano, and J. Gugenheim, "Internal hernia after laparoscopic Roux-en-Y gastric bypass for morbid obesity," Obesity Surgery, vol. 16, no. 10, pp. 1265-1271, 2006.

[6] J. M. Bellón, "Role of the new lightweight prostheses in improving hernia repair," Cirugia Espanola, vol. 85, no. 5, pp. 268-273, 2009.

[7] A. Fachinelli and M. R. Maciel Trindade, "Qualitative and quantitative evaluation of total and types I and III collagens in patients with ventral hernias," Langenbeck's Archives of Surgery, vol. 392, no. 4, pp. 459-464, 2007.

[8] E. Yahchouchy-Chouillard, T. Aura, O. Picone, J.-C. Etienne, and A. Fingerhut, "Incisional hernias: I. Related risk factors," Digestive Surgery, vol. 20, no. 1, pp. 3-9, 2003.

[9] G. Birgisson, A. E. Park, M. J. Mastrangelo, D. B. Witzke, and U. B. Chu, "Obesity and laparoscopic repair of ventral hernias," Surgical Endoscopy, vol. 15, no. 12, pp. 1419-1422, 2001.

[10] L. A. Israelsson and T. Jonsson, "Overweight and healing of midline incisions: the importance of suture technique," European Journal of Surgery, vol. 163, no. 3, pp. 175-180, 1997.

[11] A. Pans, P. Elen, W. Dewé, and C. Desaive, "Long-term results of polyglactin mesh for the prevention of incisional hernias in obese patients," World Journal of Surgery, vol. 22, no. 5, pp. 479-483, 1998.

[12] H. J. Sugerman, J. M. Kellum Jr., H. D. Reines, E. J. DeMaria, H. H. Newsome, and J. W. Lowry, "Greater risk of incisional hernia with morbidly obese than steroid-dependent patients and low recurrence with prefascial polypropylene mesh," American Journal of Surgery, vol. 171, no. 1, pp. 80-84, 1996.

[13] G. M. Eid, S. G. Mattar, G. Hamad et al., "Repair of ventral hernias in morbidly obese patients undergoing laparoscopic gastric bypass should not be deferred," Surgical Endoscopy, vol. 18, no. 2, pp. 207-210, 2004.

[14] S. S. Ching, A. I. Sarela, S. P. L. Dexter, J. D. Hayden, and M. J. McMahon, "Comparison of early outcomes for laparoscopic ventral hernia repair between nonobese and morbidly obese patient populations," Surgical Endoscopy, vol. 22, no. 10, pp. 2244-2250, 2008.

[15] I. Raftopoulos and A. P. Courcoulas, "Outcome of laparoscopic ventral hernia repair in morbidly obese patients with a body mass index exceeding $35 \mathrm{~kg} / \mathrm{m} 2$," Surgical Endoscopy, vol. 21, no. 12, pp. 2293-2297, 2007.

[16] J. Strzelczyk and L. Czupryniak, "Polypropylene mesh in prevention of postoperative hernia in bariatric surgery," Annals of Surgery, vol. 241, no. 1, pp. 196-197, 2005.

[17] F. Helgstrand, I. Gögenur, and J. Rosenberg, "Prevention of parastomal hernia by the placement of a mesh at the primary operation," Hernia, vol. 12, no. 6, pp. 577-582, 2008. 
[18] Z. Tsereteli, B. A. Pryor, B. T. Heniford, A. Park, G. Voeller, and B. J. Ramshaw, "Laparoscopic ventral hernia repair (LVHR) in morbidly obese patients," Hernia, vol. 12, no. 3, pp. 233-238, 2008.

[19] A. Saxe, S. Schwartz, L. Gallardo, E. Yassa, and A. Alghanem, "Simultaneous panniculectomy and ventral hernia repair following weight reduction after gastric bypass surgery: is it safe?" Obesity Surgery, vol. 18, no. 2, pp. 192-195, 2008.

[20] A. Iljin, D. Szymanski, J. Kruk-Jeromin, and J. Strzelczyk, "The repair of incisional hernia following Roux-en-Y gastric bypass: with or without concomitant abdominoplasty?" Obesity Surgery, vol. 18, no. 11, pp. 1387-1391, 2008. 


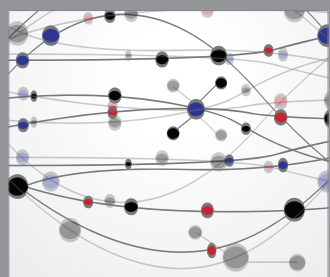

The Scientific World Journal
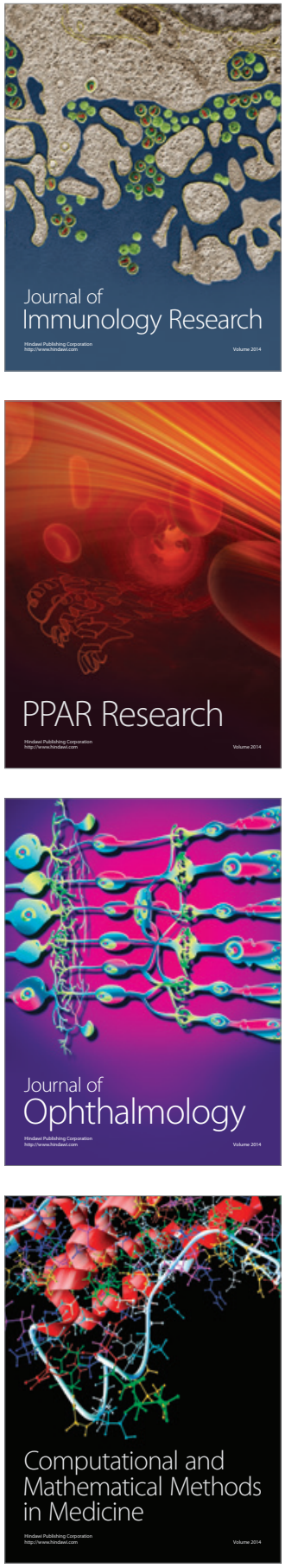

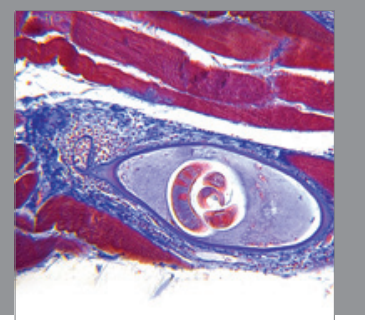

Gastroenterology

Research and Practice
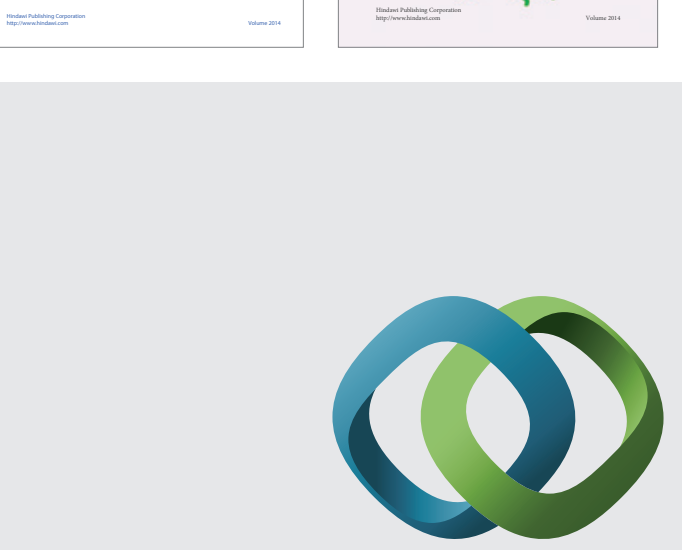

\section{Hindawi}

Submit your manuscripts at

http://www.hindawi.com
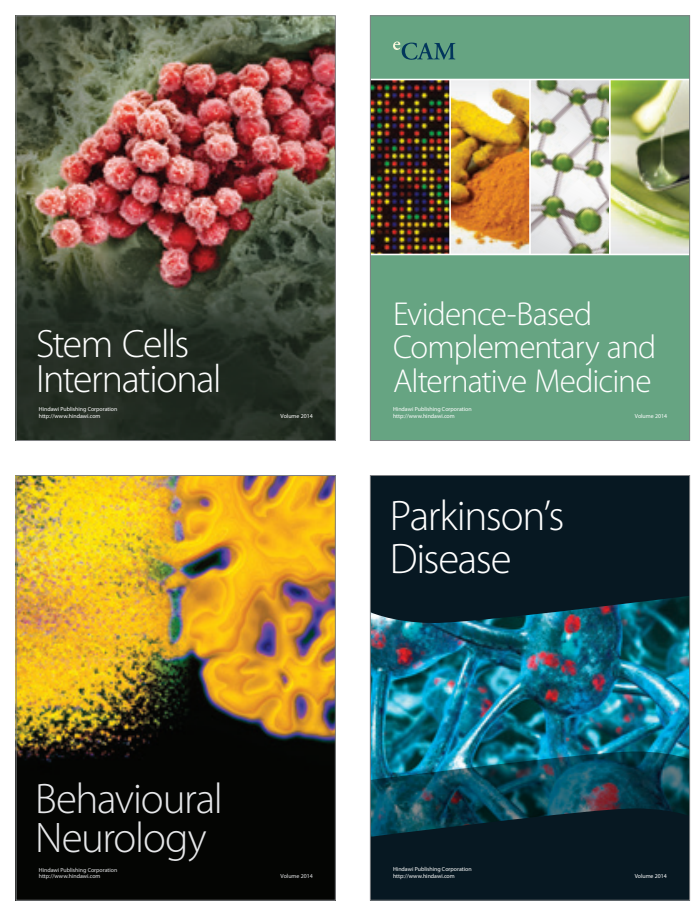

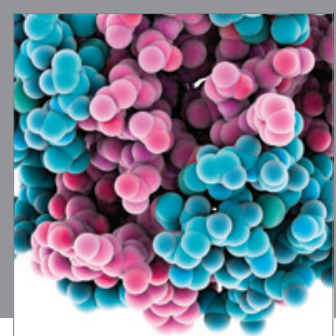

Journal of
Diabetes Research

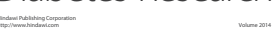

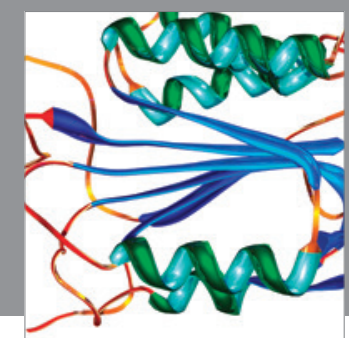

Disease Markers
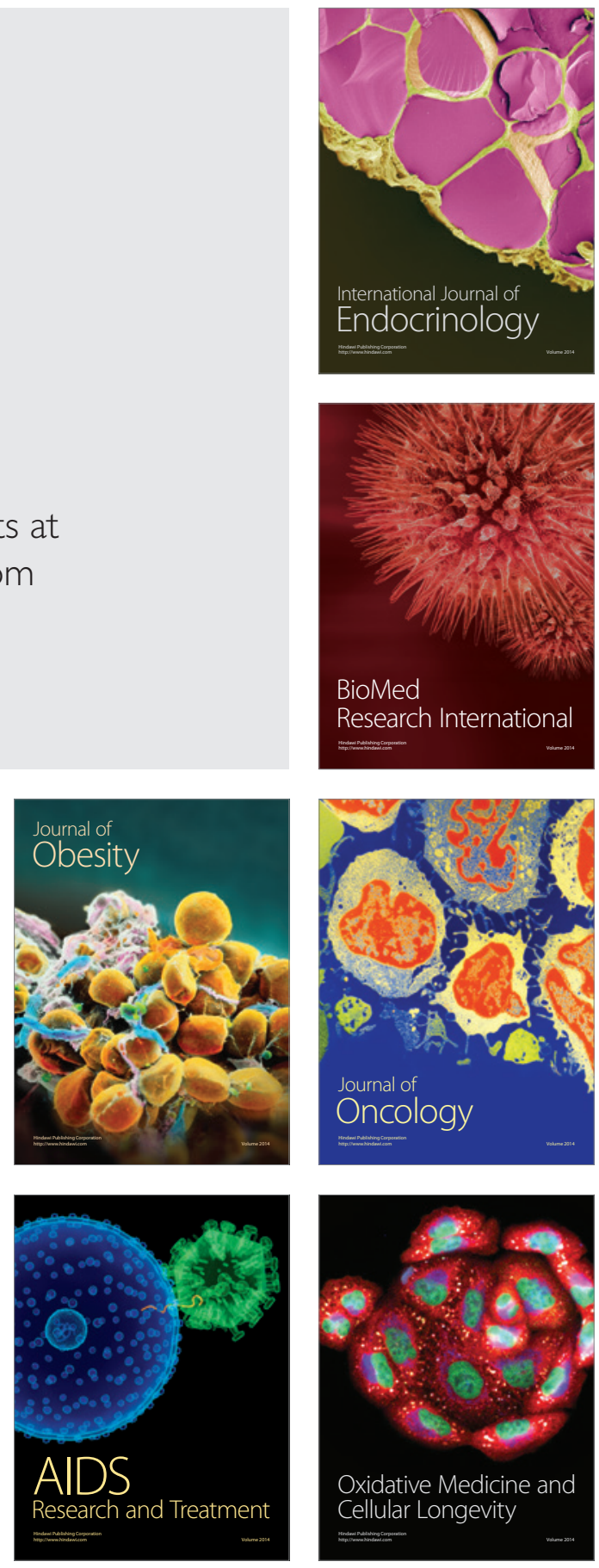